\title{
Vortices in two-dimensional superconducting weakly coupled wire networks
}

\author{
H. S. J. van der Zant* \\ Department of Electrical Engineering and Computer Science, Massachusetts Institute of Technology, Cambridge, Massachusetts 02139 \\ and Department of Applied Physics, Delft University of Technology, P.O. Box 5046, 2600 GA Delft, The Netherlands \\ M. N. Webster ${ }^{\dagger}$ \\ Department of Applied Physics, Delft University of Technology, P.O. Box 5046, 2600 GA Delft, The Netherlands \\ J. Romijn \\ Delft Institute of Micro Electronics and Submicron Technology (DIMES), Delft University of Technology, \\ P.O. Box 50532600 GB Delft, The Netherlands \\ J. E. Mooij \\ Department of Applied Physics, Delft University of Technology, P.O. Box 5046, 2600 GA Delft, The Netherlands
} (Received 26 May 1993; revised manuscript received 24 February 1994)

\begin{abstract}
We have fabricated superconducting weakly coupled wire networks made in thin amorphous niobium-silicon films of high normal-state resistivity and have investigated vortex dynamics in these networks. For the temperatures of interest, the wires have a width in the order of, and a length much larger than the Ginzburg-Landau (GL) coherence length. We find that the vortex mobility in the wire networks is much lower than in Josephson junction arrays. The ratio of the length of the single wires to the GL coherence length determines whether a Kosterlitz-Thouless-Berezinskii transition can be observed in zero field, as well as oscillations at low temperatures in the magnetoresistance. At low temperatures the critical current does not show oscillations in a magnetic field, which might be a consequence of the existence of special vortex configurations in networks with a linear current-phase relation.
\end{abstract}

\section{INTRODUCTION}

Weak two-dimensional (2D) superconducting systems exhibit a broad resistive transition around the bulk BCS transition temperature $T_{c 0}{ }^{1}$ In the critical region below $T_{c 0}$, the amplitude of the superconducting order parameter is constant over the whole sample, but the weakness of the system allows fluctuations of its phase. Even in zero magnetic field vortices may be induced thermally and a Kosterlitz-Thouless-Berezinskii (KTB) phase transition $^{2}$ is expected to occur. The KTB transition separates a resistive state with free vortices from a superconducting state below the transition where all vortices are bound in pairs. This KTB transition has been observed experimentally in $2 \mathrm{D}$ thin films with a high normal-state sheet resistance ${ }^{3}$ and in regular arrays of superconducting islands, weakly coupled to each other by Josephson junctions. ${ }^{4}$ In a perpendicular magnetic field the behavior of the discrete Josephson-junction arrays is clearly different from that of thin films: The resistance and critical current of junction arrays are periodic in a magnetic field with period $f=1$, where $f$ is the applied flux per cell divided by the flux quantum $\left(\Phi_{0}=h / 2 e\right)$, and at fractional values of $f$, minima (maxima) in the resistance (critical current) are observed. ${ }^{5}$

A 2D network of superconducting nodes coupled by small wires can be viewed as a similar system $^{3}$ as a $2 \mathrm{D}$ Josephson-junction array if the single wires are weak. It turns out that the criterion for the weakness of the wire bond is its normal-state resistance. To observe the KTB and BCS transitions separately, one needs resistances of the order of $1 \mathrm{k} \Omega$. This also applies for the sheet resistance of films or the normal-state resistance of tunnel junctions in arrays. Most experiments in wire networks, however, have been performed in samples with lowresistance wires (typically, $<1 \Omega$ ). ${ }^{6}$ In these strongly coupled networks, mean-field fluctuations of the magnitude of the order parameter are important. Only a few experiments have been performed in the weakly coupled limit. Both dynamic ac measurements ${ }^{7}$ and static dc measurements $^{8}$ have shown a KTB transition in zero magnetic field. Recently, the crossover from the strongly coupled toward the weakly coupled limit has been studied. ${ }^{9}$

Although wire networks and junction arrays can qualitatively be viewed as similar systems, it is not a priori clear that measurable properties such as the resistive response will be the same. Wire networks are made in films with high normal-state resistivities, and as a consequence, the Ginzburg-Landau (GL) coherence length ( $\xi$ ) is small, typically in the order of $20 \mathrm{~nm}$ at the lowest temperatures. For 2D wire networks, fabricated with the present technology, the width of the single wires cannot be made significantly smaller than $\xi$. For well-defined wires, the length will be larger than $\xi$. In that case, the supercurrent through a wire depends linearly on the phase difference. In contrast, Josephson-junction arrays have a sinusoidal current-phase relation for the bonds. One could therefore argue that the behaviors of wire networks and junctions arrays are comparable, as long as the 
phase differences between adjacent nodes $(\Delta \phi)$ are small, i.e., when $\sin (\Delta \phi) \approx \Delta \phi$. The zero-field-KTB transition involves long-range logarithmic vortex interactions where phase differences between adjacent nodes are small. Only the core energy is higher for wires with the same resistance. In contrast, in the dynamics we expect strong differences. Examples are when vortices, moving from one cell to the next, cross the single wires of the network (this process determines the vortex mobility) and when the critical current is measured. As yet, there has been no systematic study on wire networks to verify this picture and to explore the consequences of the linear current-phase relation of wire networks.

In this paper we present results of a detailed experimental study on the resistive transition and on the behavior in a magnetic field of weakly coupled wire networks. We investigate the zero-field-KTB transition, the vortex mobility, the magnetoresistance, and the critical current measured as a function of applied magnetic field. The data on the wire networks are compared with similar data on our Josephson-junction arrays. ${ }^{4,5}$ The KTB transition looks qualitatively very similar to the one of junction arrays, but quantitatively one has to take into account a much lower vortex mobility of wire networks. A clear difference is found in the critical current measured versus applied field; in wire networks, we do not observe oscillations at the lowest temperatures, whereas in junction arrays always oscillations are found.

The paper is organized as follows. In the next section we find the effective coupling energy, the vortex mobility, and the flux-flow resistance of wire networks. We show that the vortex mobility in wire networks is generally much smaller than in junction arrays. In Sec. III we describe the fabrication of the amorphous NbSi layers and of the wire networks made in these films. Section IV deals with the experimental results. First, we determine the normal-state resistance and the BCS transition temperature from the resistive transition. Then the experimental results on the magnetoresistance, on the critical current in a magnetic field, on the linear resistance as a function of temperature in zero and nonzero field, and on the nonlinear resistance are discussed. In Sec. V the experimental results are evaluated, and in Sec. VI we give our conclusions.

\section{WIRE NETWORKS VERSUS JOSEPHSON-JUNCTION ARRAYS}

\section{A. Basic properties}

We consider a square 2D network of nodes which are weakly connected. Following Teitel and Jayaprakash, ${ }^{10}$ in the constant-amplitude approximation the interaction energy $E_{n m}$ between two adjacent nodes $n$ and $m$ can be written as

$$
E_{n m}=-J^{*} V\left(\phi_{n m}\right),
$$

where $J^{*}$ is the coupling constant, $V\left(\phi_{n m}\right)$ is some potential periodic in $2 \pi$, and $\phi_{n m}$ is the gauge-invariant phase difference across nodes $n$ and $m, \phi_{n m}=\left(\phi_{n}-\phi_{m}-A_{n m}\right)$. The $\phi_{n}$ and $\phi_{m}$ are the phases of the order parameter on nodes $n$ and $m$ respectively, and $A_{n m}$ is the line integral of the vector potential $\mathbf{A}$ :

$$
A_{n m}=\left(2 \pi / \Phi_{0}\right) \int_{n}^{m} \mathbf{A} d \boldsymbol{l} .
$$

In every cell of the network, summation of the $A_{n m}$ 's must give the applied flux, i.e.,

$$
\sum_{\text {cell }} A_{n m}=\frac{2 \pi B S}{\Phi_{0}}=2 \pi f,
$$

where $B$ is the perpendicular applied magnetic field, $S$ the area of a cell in the network, and $f$ is the frustration index.

The interaction energy can be calculated ${ }^{3}$ from the supercurrent $\left(i_{s}\right)$-phase $(\Delta \phi)$ relation

$$
E_{n m}=-\left(\Phi_{0} / 2 \pi\right) \int_{0}^{\phi_{n m}} i_{s}(\Delta \phi) d \Delta \phi
$$

For Josephson-junction arrays, the single junctions have a sinusoidal current-phase relation and $E_{n m}=$ $-E_{J}(T) \cos \left(\phi_{n m}\right)$, where $E_{J}(T)$ is the temperaturedependent Josephson coupling energy. For small phase differences one can approximate the interaction energy of junction arrays by $E_{n m} \approx-\frac{1}{2} E_{J}(T)\left(\phi_{n m}\right)^{2}$. In a $2 \mathrm{D}$ wire network the current-phase relation of the single wires depends on the size of the GL coherence length relative to $s$, the length of the wires. For $s \leq \xi(T)$, the current-phase relation is sinusoidal and the coupling is Josephsonlike. For $s \gg \xi(T)$, the current-phase relation is a set of straight lines, separated by $2 \pi$ in $\phi_{n m}$. In this case the supercurrent is given by

$$
i_{s}=i_{c}(T) \frac{\phi_{n m} \pm 2 \pi k}{\Delta \phi_{\max }}
$$

where $i_{c}(T)$ is the Ginzburg-Landau depairing current of a single wire, $\Delta \phi_{\max }$ is the maximum phase difference across adjacent nodes, $\Delta \phi_{\max }=\left(\frac{2}{3} \sqrt{3}\right) s / \xi(T)$, and $k$ is an integer. With Eqs. (3) and (4), one finds that $E_{n m}=-\frac{1}{2} J(T)\left\{\phi_{n m} \pm 2 \pi k\right\}^{2}$, which defines the wire network equivalent of the Josephson coupling energy

$$
J(T)=\frac{3 \sqrt{3} \xi(T)}{2 s} \frac{\Phi_{0}}{2 \pi} i_{c}(T) .
$$

To compensate for the effects of the temperature dependence of the coupling energy, a normalized temperature $\tau$ is introduced, defined as

$$
\tau=\frac{k_{B} T}{J(T)} .
$$

\section{B. Resistive behavior}

Resistance in 2D weakly coupled overdamped systems is generally ascribed to vortex motion across the sample width. For viscous, uncorrelated vortex motion, the network resistance $R(T)$ is determined by the 2D density of free vortices $n_{f}(T)$ and their mobility $\mu^{\prime}(T)$ and is given by $R(T)=c \Phi_{0}^{2} n_{f}(T) \mu^{\prime}(T)$, where $c$ is a constant. It is convenient to define an effective vortex mobility as $\mu(T)=\mu^{\prime}(T) \Phi_{0}^{2} /\left(R_{n} S\right)$, with $R_{n}$ the normal-state resis- 
tance. Then the flux-flow resistance can be written as

$$
\frac{R}{R_{n}}=c S n_{f}(T) \mu(T) \text {. }
$$

In zero field, free vortices may be present. They can be excited by thermally activated dissociation of vortexantivortex pairs (above the KTB transition) or by current-induced vortex pair unbinding (above and below the KTB transition). Expressions for $n_{f}(T)$ have been given by Halperin and Nelson. ${ }^{11}$ In finite systems, below the transition temperature there is always a probability for a thermally activated vortex to cross the sample. ${ }^{4}$ In zero field, the energy barrier for this process of singlevortex crossings is

$$
U_{2 \mathrm{D}}=E_{\text {rore }}+\pi E_{J}(T) \ln \{2 W / \pi\},
$$

where $W$ is the width of the array expressed in units of the lattice constant $s$ and where $E_{\text {core }}$ is the core energy of a single vortex. In a Josephson-junction array, $E_{\text {core }} \approx 4 E_{J}(T)$.

With a magnetic field applied perpendicular to the sample, magnetically induced vortices enter the finite sample above a certain entry field (typically of order $f \approx 1 / W)$ and $U_{2 \mathrm{D}}$ is dramatically lower than the zerofield value. ${ }^{4}$ Above this entry frustration and below the KTB transition temperature, the density of free vortices is $B / \Phi_{0}=f / S$ and the resistance is expected to follow the temperature dependence of the effective vortex mobility. However, for fractional values of $f$ (e.g., $\frac{1}{2}, \frac{1}{3}$, and $\frac{2}{5}$ ), the vortices may form a lattice which is pinned on the underlying network.

In the remainder of this section, we calculate the vortex mobility for single independent vortices. In $2 \mathrm{D}$ superconducting networks, the vortex mobility is determined by the energy barrier $U_{\text {bar }}$ for a vortex to cross from one cell to the next. In junction arrays, the barrier is determined by the change in energy associated with phase differences between adjacent nodes when a vortex moves from one cell to the next. The barrier height has been calculated numerically by Lobb, Abraham, and Tinkham. ${ }^{12}$ Their result for a large square array is $U_{\mathrm{bar}}=0.2 E_{J}(T)$. The probability to cross a single junction is derived by using the result for phase slip in a single overdamped junction, which yields $\mu(T)$ $=I_{0}^{-2}\{1 / 10 \tau\}$. Here $I_{0}$ is the zero-order Bessel function with argument $1 / 10 \tau$. Near the KTB transition temperature $\left(\tau_{\mathrm{KTB}}=0.95\right), \mu \approx 1$, indicating that single vortices can move freely in the periodic lattice.

In wire networks, there is a similar kinetic energy ( $U_{\text {kin }}$ ) contribution to the energy barrier. However, there is an additional, additive contribution to the energy barrier which is connected to the fact that when a vortex crosses a single wire, the amplitude of the order parameter vanishes locally. This process yields a loss of condensation energy. For small wires with $w<\xi(T)$, this second term can be estimated from the barrier $U_{1 \mathrm{D}}$ against phase slip in a one-dimensional wire, ${ }^{13}$

$$
U_{1 \mathrm{D}}=\frac{4 \sqrt{2}}{3} \mu_{0} H_{c}^{2} w d \xi(T)=0.94 \frac{s}{\xi(T)} J(T),
$$

where $H_{c}$ is the thermodynamic critical field. The total energy barrier for a vortex to cross from one cell to the next is the sum of the two contributions,

$$
U_{\mathrm{bar}}=U_{\mathrm{kin}}+0.94 \frac{s}{\xi(T)} J(T)
$$

We previously estimated $U_{\text {kin }}$ to be $0.2 J(T)$. Recent calculations ${ }^{9}$ have shown that the kinetic energy contribution for small fields is equal to $-2.46 J(T)$. For our networks well below $T_{c 0}, s \gg \xi(T)$ and the dominant contribution to the energy barrier comes from the loss of condensation energy, the second term in Eq. (10). For the fields of interest, this term is frustration independent.

For wire networks, there is no reason to use the Bessel-function dependence of the Josephson-junction arrays. It seems reasonable to use the Boltzmann expression for thermal activation, and the effective vortex mobility is

$$
\mu(T)=\exp \left\{-U_{\mathrm{bar}} / k_{B} T\right\}=\exp \left\{-u_{\mathrm{bar}} / \tau\right\},
$$

where $u_{\text {bar }}=-2.46+0.94 \mathrm{~s} / \xi(T)$. Comparing this to the result for Josephson-junction arrays, one sees that vortices in our wire networks are stronger pinned in the intrinsic periodic network potential than vortices in junction arrays. Even at temperatures near the KTB phase transition, the vortex mobility in our networks is orders of magnitude lower than in Josephson-junction arrays.

Strictly speaking, our single wires are not one dimensional, but have a $\xi(T)$ in the order of the single wire width. We assume that Eq. (10) is still a reasonable approximation of the energy barrier. For very wide wires the energy barrier is determined by the energy barrier for a vortex to cross a $2 \mathrm{D}$ superconducting system. In zero field the energy barrier is given in Eq. (8).

\section{SAMPLE FABRICATION}

Networks are patterned in niobium-silicon NbSi films, which have a high normal-state resistance. Films are obtained with a cosputter technique; i.e., the oxidized silicon substrates rotate under $\mathrm{Nb}$ and $\mathrm{Si}$ targets. $\mathrm{Nb}$ is dc and $\mathrm{Si}$ rf magnetron sputtered in an argon pressure of 1 $\mathrm{Pa}$. The background pressure before sputtering is typically $3 \times 10^{-5} \mathrm{~Pa}$. Deposition is carried out at room temperature. The substrates rotate with 23.4 cycles per minute, and per cycle about one monolayer $\mathrm{NbSi}$ is deposited. Two series with different $\mathrm{NbSi}$ composition have been made by changing the power for the Si deposition. The deposition rate for $\mathrm{Nb}$ is kept constant at 1.7 $\mathrm{nm} / \mathrm{min}$. The rates for $\mathrm{Si}$ deposition are either 2.7 $\mathrm{nm} / \mathrm{min}$ (series A) or $6.7 \mathrm{~nm} / \mathrm{min}$ (series B). Series A contains $70 \% \mathrm{Nb}$ and series B $42 \%$. By varying the deposition time, films with different thicknesses have been obtained. We have examined several films with $\mathrm{x}$ ray diffraction and high-resolution transmission electron microscopy (TEM). X-ray diffraction shows no indication for the formation of separate layers of $\mathrm{Nb}$ or $\mathrm{Si}$. In the TEM photographs no ordering at all is found in a film of $3 \mathrm{~nm}$ thickness. In a film of $d=10 \mathrm{~nm}$, only some weak short-range order on a scale less than $1 \mathrm{~nm}$ is observed. We conclude therefore that our films are amor- 
TABLE I. Parameters of the four wire networks studied. $R_{\mathrm{sqr}}$ is the normal-state sheet resistance of the film measured at $4.2 \mathrm{~K}, d$ the thickness, $\rho$ the resistivity (measured at $4.2 \mathrm{~K}$ ), and $l$ the mean free path of the films. $R_{n}$ is the normal-state network resistance, $T_{c 0}$ the mean-field BCS transition temperature, $r_{n}$ the resistance of a single wire, $w$ the width of a single wire, $\xi_{0}$ the BCS coherence length, $\xi_{\mathrm{GL}}(0)$ the zero-temperature Ginzburg-Landau coherence length, $s$ the length of a single wire, and $L / W$ is the length/width ratio of the network.

\begin{tabular}{|c|c|c|c|c|c|c|c|c|c|c|c|c|}
\hline Network & $\begin{array}{l}R_{\text {sqr }} \\
(\Omega) \\
\end{array}$ & $\begin{array}{c}d \\
(\mathrm{~nm})\end{array}$ & $\begin{array}{c}\rho \\
(\mu \Omega \mathrm{m})\end{array}$ & $\begin{array}{c}l \\
(\mathrm{~nm})\end{array}$ & $\begin{array}{l}R_{n} \\
(\Omega) \\
\end{array}$ & $\begin{array}{l}T_{c 0} \\
(\mathbf{K})\end{array}$ & $\begin{array}{c}r_{n} \\
(\Omega)\end{array}$ & $\begin{array}{c}w \\
(\mathrm{~nm})\end{array}$ & $\begin{array}{c}\xi_{0} \\
(\mathrm{~nm})\end{array}$ & $\begin{array}{c}\xi_{G L}(0) \\
(\mathrm{nm})\end{array}$ & $\begin{array}{c}s \\
(\mathrm{~nm})\end{array}$ & $L / W$ \\
\hline 1 & 238 & 10 & 238 & 0.4 & 2450 & 2.135 & 1250 & 97 & 840 & 16 & 500 & 2 \\
\hline 2 & 238 & 10 & 238 & 0.4 & 1265 & 2.16 & 633 & 190 & 850 & 16 & 500 & 2 \\
\hline 3 & 100 & 20 & 200 & 0.5 & 2600 & 1.63 & 1520 & 154 & 1100 & 20 & 2000 & 1.71 \\
\hline 4 & 231 & 14 & 323 & 0.3 & 2350 & 1.705 & 1374 & 430 & 1050 & 15 & 2000 & 1.71 \\
\hline
\end{tabular}

phous. A similar result has been reported by others, ${ }^{14}$ who find that $\mathrm{NbSi}$ films fabricated at room temperature are amorphous if they consist of $10 \%$ or more Si. The fact that our films are amorphous is a great advantage over granular films, which consist of superconducting islands coupled by irregular Josephson junctions.

In this paper, we report on four arrays, which are made in different films. In Table I we list the BCS transition temperature $T_{c 0}$, the normal-state sheet resistance $R_{\text {sqr }}$ at $4.2 \mathrm{~K}$, the thickness $d$, and the normal-state resistivity $\rho$ of the films. If we assume a $\rho l$ product of $10^{-15}$ $\Omega \mathrm{m}^{2}$, the mean free path is of the order of $0.5 \mathrm{~nm}$. More details about the films have been published elsewhere. ${ }^{15}$

Networks are fabricated using a two-layer resist system of $100-\mathrm{nm}$ high-resolution silicon-based negative resist (SNR) on top of $125-\mathrm{nm}$ hard-baked $\left(200^{\circ} \mathrm{C}\right) \mathrm{AZ}-1470$ photoresist. Patterns are made by direct writing in the SNR resist with an electron-beam pattern generator. After developing, the pattern is transferred into the $\mathrm{AZ}$ resist and the $\mathrm{NbSi}$ films by reactive ion etching in $\mathrm{O}_{2}$ and $\mathrm{SF}_{6}$ plasmas successively. In Fig. 1 a scanning electron microscope (SEM) photograph of a completed network is shown. For this network the width of the single wires is about $100 \mathrm{~nm}$. The lattice spacing $s=500 \mathrm{~nm}$.
These networks with $s=500 \mathrm{~nm}$ have a length of 200 unit cells, $L=200$, and have a width $W$ of 99 unit cells. We have also fabricated networks with a lattice spacing of $2000 \mathrm{~nm}$. These networks have $L=180$ and $W=105$. On opposite ends, all networks are connected to $\mathrm{NbSi}$ pads over the full width, which are used to inject the current into the array along parallels, as illustrated in Fig. 2. Voltage probes are connected to the array at six places and have a width of $500 \mathrm{~nm}$. With these probes the longitudinal as well as the transverse "Hall" resistance can be measured.

\section{EXPERIMENTAL DATA}

Measurements are performed in a vacuum can placed in a standard $\mathrm{He}-4$ cryostat. Inside the vacuum can a perpendicular magnetic field can be applied by means of two coils of superconducting wire, arranged in a Helmholtz configuration. Superconducting lead and $\mu$ metal magnetic shields are placed outside the vacuum can. One flux quantum per unit cell, $f=1$, corresponds to a magnetic field of $8.28 \mathrm{mT}$ for the networks with $s=500 \mathrm{~nm}$ and to a magnetic field of $0.52 \mathrm{mT}$ for the networks with $s=2000 \mathrm{~nm}$. The maximum field that can be

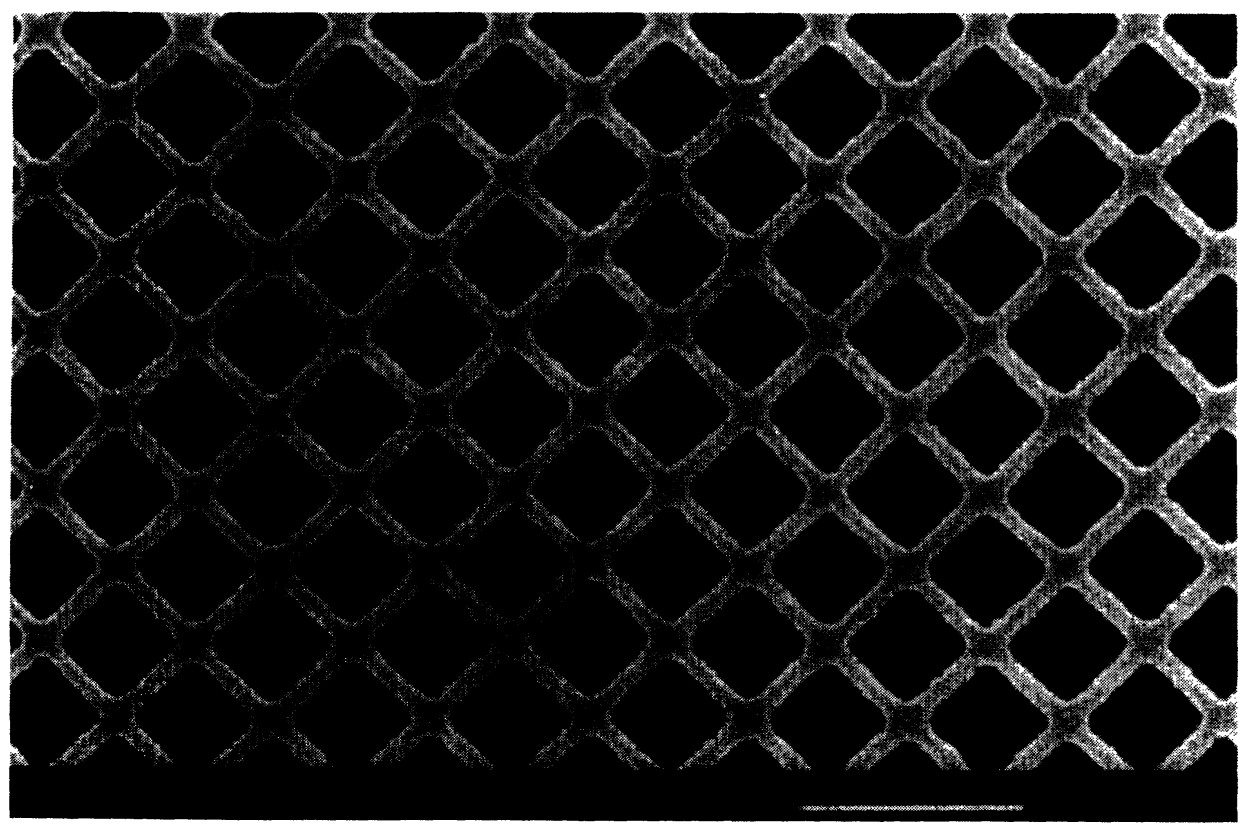

FIG. 1. Scanning electron microscope (SEM) micrograph of network 1. The white bar at the bottom indicates $1 \mu \mathrm{m}$. The width of the single wires is about $100 \mathrm{~nm}$; the lattice spacing $s$ is $500 \mathrm{~nm}$. 


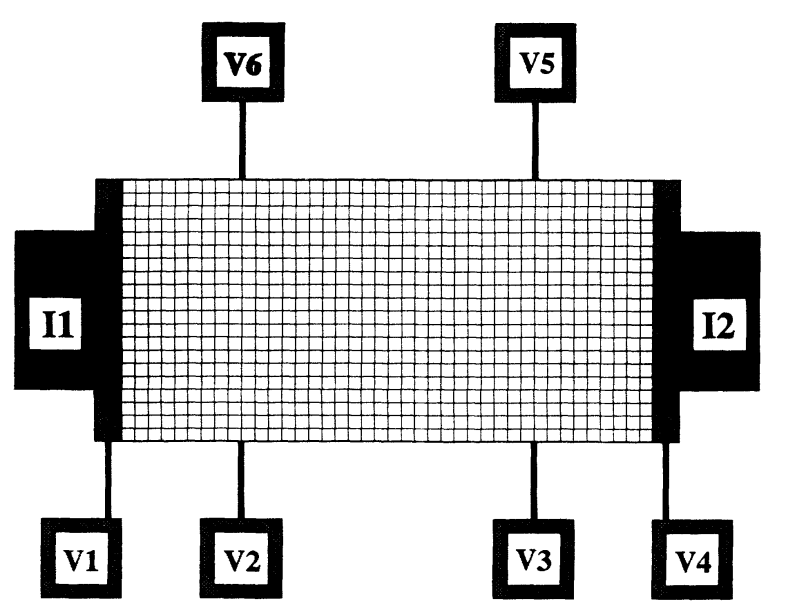

FIG. 2. Schematic drawing of a wire network.

applied is $0.13 \mathrm{~T}$, which corresponds to $f=15.7$ and 250 , respectively. Experiments are done with standard measuring equipment. The linear resistance is measured with a sinusoidal wave of $108 \mathrm{~Hz}$ and the nonlinear resistance with a square wave of $27 \mathrm{~Hz}$.

\section{A. Sample characterization}

The normal-state network resistance $R_{n}$ and the mean-field BCS transition temperature $T_{c 0}$ can be determined from the $R(T)$ curves in the high-temperature region above $T_{c 0}$. Here the resistance is dominated by fluctuations of the superconducting order parameter, which is described by the theory of Aslamazov and Larkin. ${ }^{16}$ The theoretical expression is

$$
\frac{1}{R(T)}=\frac{1}{R_{n}}+\frac{W}{L} \frac{t}{T_{c 0} R_{0}},
$$

where $t=T_{c 0}-T$ and $R_{0}=16 h / e^{2}=6.58 \times 10^{4} \Omega$. Equation (12) is valid for two-dimensional superconductors, i.e., for samples where the characteristic length for fluctuations of the order parameter is smaller than $w$. To estimate this characteristic length, we use the expression ${ }^{13}$ for the Ginzburg-Landau coherence length for temperatures above $T_{c 0}$. For network 1, Eq. (12) should be valid for temperatures $T>2.185 \mathrm{~K}$.

In Fig. 3 we show the resistive transition of network 1. We have fitted Eq. (12) to the experimental data using the procedure of Fiory, Hebard, and Glaberson. ${ }^{17}$ For different values of $R_{n}$, one plots $1 /\left[R^{-1}(T)+R_{n}^{-1}\right]$ against $T$. A straight line is found when the right value of $R_{n}$ is used. In the inset of Fig. 3, we show the result of this fit with $R_{n}=2450 \Omega$. $T_{c 0}$ is the temperature where the straight line intersects the $T$ axis, $T_{c 0}=2.135 \mathrm{~K}$. From the slope of the dashed line, we find $R_{0}=8.6 \times 10^{4}$ $\Omega$, somewhat larger than the theoretical value of $6.58 \times 10^{4} \Omega$. Results for $R_{n}$ and $T_{c 0}$ of the other samples are listed in Table I. For all samples, we find $R_{0}$ values which are somewhat larger than the theoretical value.

From the experimentally determined values of $R_{n}$, the resistances of the single wires, $r_{n}=R_{n} W / L$, and the

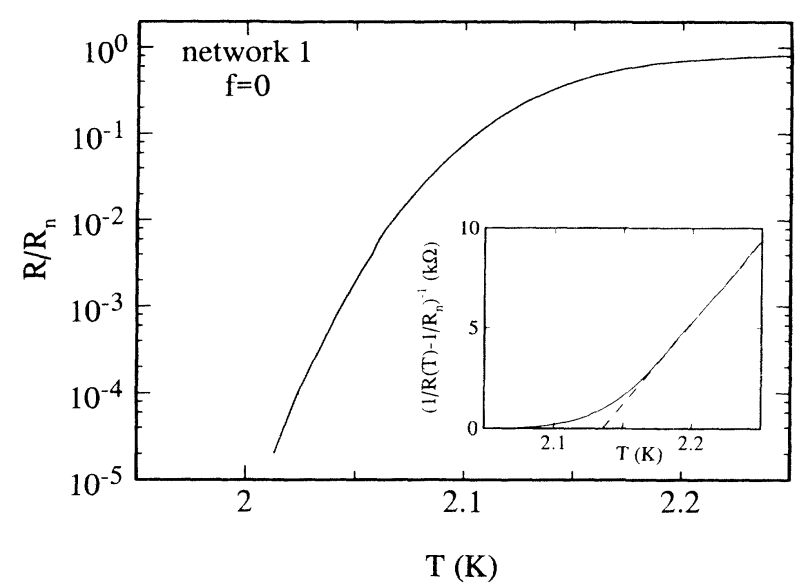

FIG. 3. Longitudinal zero-field resistance of network 1 as a function of temperature. In the inset, the inverse excess conductance is plotted as a function of temperature. From a 2D Aslamazov-Larkin fit (dashed line), the normal-state resistance and the mean-field BCS transition temperature are obtained.

widths of the single wires, $w=R_{\mathrm{sqr}} L s /\left(R_{n} W\right)$, have been calculated. For network 1 , we find $r_{n}=1225 \Omega$ and $w=97 \mathrm{~nm}$, which is in good agreement with the value obtained from the SEM photograph (Fig. 1). With the values of $T_{c 0}$, the BCS coherence length $\xi_{0} \approx 0.180 h v_{F} /\left(k_{B} T_{c 0}\right)$ can be calculated, where the Fermi velocity is taken to be $v_{F} \approx 1.3 \times 10^{6} \mathrm{~m} / \mathrm{s}$. For network 1 , we find $\xi_{0}=840 \mathrm{~nm}$. Using this value, the zerotemperature GL coherence length $\xi(0)$ is $15 \mathrm{~nm}$. In the temperature range $200<t<10 \mathrm{mK}, \xi(T)$ varies from 50 to $230 \mathrm{~nm}$ for this network.

In zero magnetic field, the array critical current $I_{c}$ is given by $(W+1) i_{c}$, where the single-wire critical current $i_{c}$ is determined by the depairing current $i_{0}$ via $i_{c}=2 i_{0} / 3 \sqrt{3}$ with $i_{0}=\pi \Delta^{2}(T) s / 4 e k_{B} \operatorname{Tr}_{n} \xi(T)$ and $\Delta(T)$ the BCS gap. Using $\Delta^{2}(T)=9.4 k_{B}^{2} T_{c 0} t$, one finds, for $T \approx T_{c 0}$,

$$
I_{c}(T)=(W+1) i_{c 0} t^{3 / 2},
$$

with

$$
i_{c 0}=\frac{3.33 k_{B} s}{e r_{n}}\left[\frac{1}{T_{c 0} \xi_{0} l}\right]^{1 / 2} .
$$

We have measured $I_{c}$ as a function of $T$. At low temperatures, the voltage switches to a finite value and there is no problem in defining $I_{c}$. At higher temperatures $t<50$ $\mathrm{mK}$, the $I-V$ curve is a smooth curve and $I_{c}$ is defined as the current where $V=500 \mathrm{nV}$. In Fig. 4 we show $I_{c}^{2 / 3}(T)$ of network 1 as a function of temperature in the range $t<400 \mathrm{mK}$. A straight line is found which intersects the $T$ axis at $2.13 \mathrm{~K}$. The measured values are $30 \%$ lower than predicted by Eq. (13). Given the estimations in determining $\xi_{0}$ and $l$, this is a very satisfactory agreement. For the other arrays, we again found the temperature dependence of Eq. (13) for temperatures near $T_{c 0}$ and absolute values of $I_{c}(T)$ close to the values predicted by Eq. (13). With Eqs. (6) and (13), the $\tau$ scale can be ex- 


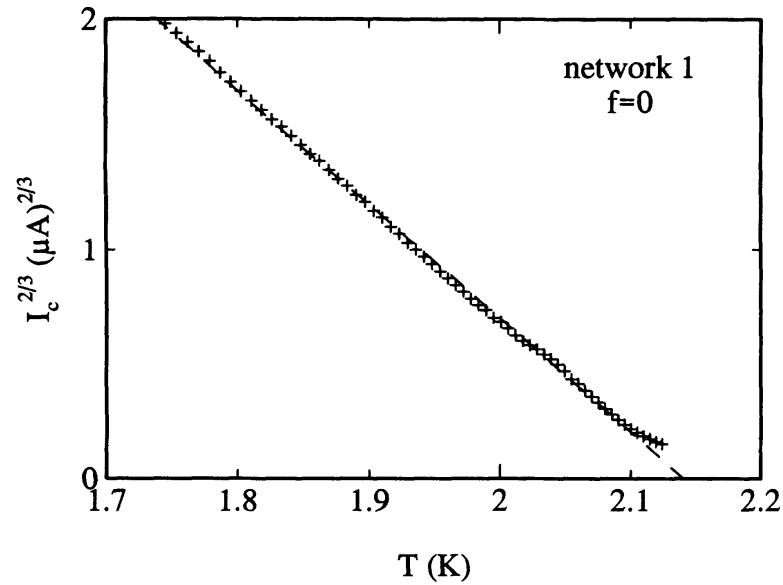

FIG. 4. Zero-field critical current of network 1 to the power $\frac{2}{3}$ as a function of temperature. The dashed line is a fit to Eq. (13).

pressed in measurable quantities,

$$
\tau=c_{1} \frac{r_{n} T^{2}}{t T_{c 0}}
$$

where $c_{1}=6.81 \times 10^{-5} \Omega^{-1}$.

Knowing the critical current, the contribution of the self-induced magnetic flux can be calculated. For network 1 at the KTB transition temperature $(1.985 \mathrm{~K}$; see next section), $i_{c}=260 \mathrm{nA}$. We estimate the geometrical inductance of one cell $L_{\text {cell }}$ to be $1 \mathrm{pH}$. With these numbers, the self-induced magnetic flux $L_{\text {cell }} i_{c}=2.7 \times 10^{-19}$ $\mathrm{W} \mathrm{\textrm {m } ^ { 2 }}$, which is four orders of magnitude smaller than $\Phi_{0}$. This is consistent with the fact that the penetration depth for flux (at the KTB transition temperature given by the universal value ${ }^{3}$ of $2 \mathrm{~cm} \mathrm{~K}^{-1}$ ) is much larger than the network size $(50 \mu \mathrm{m} \times 150 \mu \mathrm{m})$. Therefore, in our weakly coupled networks, the magnetic field is uniform over the whole network area and equal to the applied field.

\section{B. Magnetic field behavior}

We give results of the network linear resistance and the network critical current, measured as a function of frustration at various temperatures. The resistance is measured using a small current with an amplitude of $0.1 \mathrm{nA}$ (rms) per single wire. The critical current has been defined as the current where $V=500 \mathrm{nV}$. In Fig. 5 the longitudinal array resistance of network 1 is shown. From $a$ to $g$, temperature decreases. Curves $a, b$, and $c$ are measured above $T_{c 0}$. For all curves, the temperature is above the zero-field-KTB transitions temperature of $1.985 \mathrm{~K}$. The first oscillations with a period of $f=1$ become visible in curve $d$. With decreasing temperature, the oscillations are more pronounced and are found at higher values of $f$. Curves are highly symmetric around $f=0$. For curve $g$, oscillations are seen up to the maximum frustration of $f=16$. The oscillations are superimposed on a parabolic background.

We also find oscillations for the other network with $s=500 \mathrm{~nm}$. Again, oscillations become visible just below $T_{c 0}$. However, at low temperatures, clear oscillations are

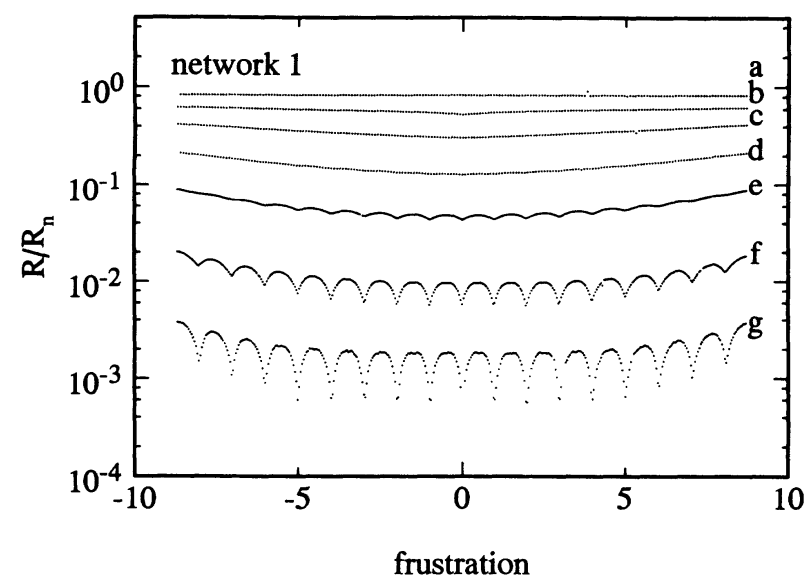

FIG. 5. Normalized longitudinal resistance of network 1 vs frustration for seven different temperatures with (a) $T=2.3 \mathrm{~K}$, (b) $T=2.19 \mathrm{~K}$, (c) $T=2.16 \mathrm{~K}$, (d) $T=2.12 \mathrm{~K}$, (e) $T=2.09 \mathrm{~K}$, (f) $T=2.06 \mathrm{~K}$, and (g) $T=2.03 \mathrm{~K}$.

only found up to $f \approx 10$. For this array, the parabolic background is much more pronounced. Around $f=0$, no oscillations at all are observed in the networks with $s=2000 \mathrm{~nm}$. In network 3 , at $60 \mathrm{mK}$ below $T_{c 0}$ small oscillations become visible in the region $-50<f<-33$ and $33<f<50$. Here the resistance varies between 0.05 and $0.2 \Omega$. For the networks with $s=2000 \mathrm{~nm}$, a more complicated background signal is observed. For small fields $f<25$, there is a parabolic dependence of $R$ on $f$. For $f \approx 33$ the resistance shows a local maximum, and at $f \approx 40$ there is a local minimum. For $f>40$ the resistance keeps on increasing up to $f=250$.

In Fig. 6(a) we give the network resistance for network 1 on an expanded scale. The temperature is $125 \mathrm{mK}$ below $T_{c 0}$, and the normalized temperature $\tau=1.3$. The resistances at $f=0$ and \pm 1 are almost equal and are about one order of magnitude smaller than the maximum resistance at $f \approx 0.4$. For network 2 , the resistance is shown in Fig. 6(b). Here the temperature is $60 \mathrm{mK}$ below $T_{c 0}$ and $\tau=6.8$. Although the effective temperature is higher, the resistances in Fig. 6(b) are lower by a factor of 10 compared to the curve in Fig. 6(a). In Fig. 6(b) we again find that the resistance at $f=0$ is one order of magnitude smaller than at $f=0.4$. The resistance at $f=1$ is 5 times larger than at $f=0$ (more pronounced parabolic background for this array). A variety of structure is visible in both figures. Dips in the resistance are present at $f= \pm \frac{1}{2}$ and smaller ones at $f= \pm \frac{1}{3}$ and $\pm \frac{2}{3}$.

We have also measured the transverse voltage across probes 2 and 6 (see Fig. 2) as a function of temperature. At $T=4.2 \mathrm{~K}$, the resistance is 10 times smaller than the longitudinal resistance measured between probes 1 and 4 . Lowering the temperature, the transverse resistance follows the temperature dependence of the longitudinal resistance. As a function of $f$ around $f=0$ within measuring accuracy, there is no contribution to the transverse voltage of an odd magnetic field term. We find that $R_{\text {Hall }} / R_{n, \text { long }}<10^{-4}$. This means that vortices move in the direction perpendicular to the applied transport current. 


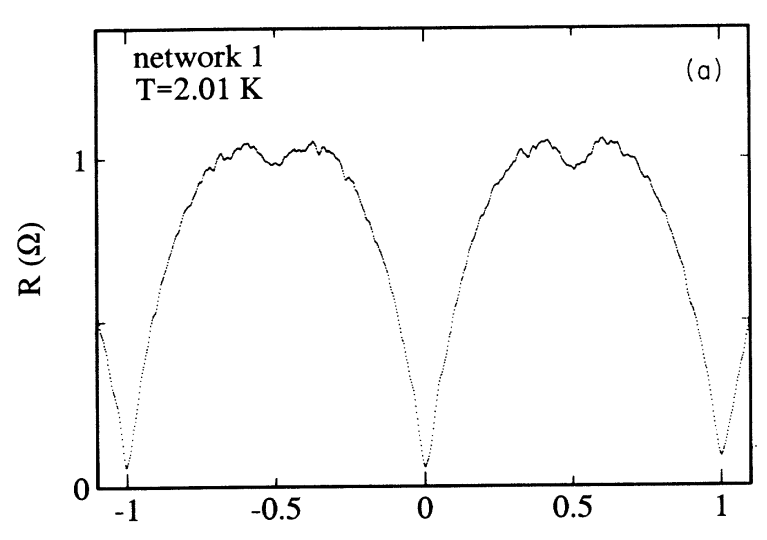

frustration

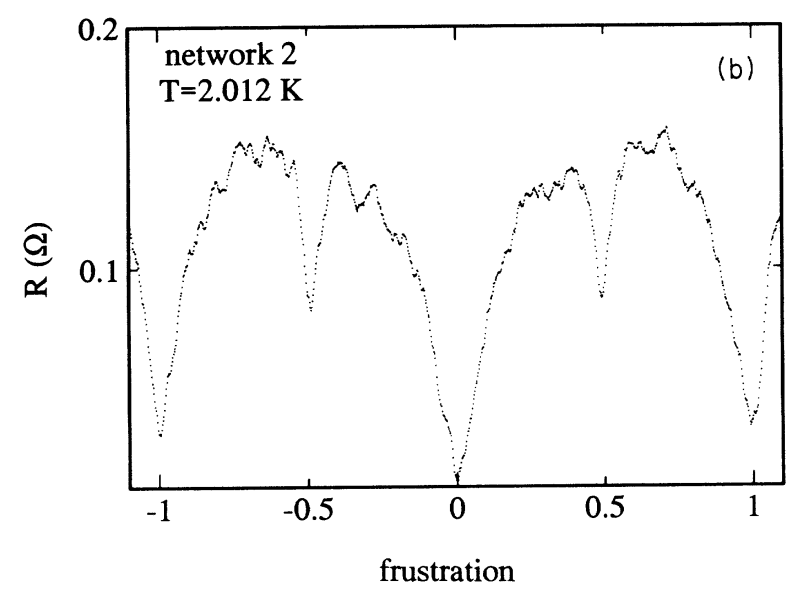

FIG. 6. (a) Longitudinal resistance of network 1 as a function of frustration on an expanded scale measured at a temperature of $2.01 \mathrm{~K}(\tau=1.3)$. (b) Longitudinal resistance of network 1 as a function of frustration on an expanded scale measured at a temperature of $2.012 \mathrm{~K}(\tau=6.8)$.

Next, we show the critical current $I_{c}$ as a function of $f$. Detailed measurements were only performed on network 1. For four different temperatures, the result is shown in Fig. 7. In the range $15<t<95 \mathrm{mK}$, small oscillations are observed, yet there is no fine structure at fractional values of $f$. Below $2.04 \mathrm{~K}(t=95 \mathrm{mK})$, there are no oscillations visible in the array critical current and only a parabolic dependence on the magnetic field remains. This is a surprising result, since in the network resistance still oscillations are seen (curve $g$ in Fig. 5).

For network 1, we have analyzed the parabolic dependence of both the resistance and the critical current in more detail. We have found reasonable agreement with a model which takes into account the reduction of $T_{c 0}$ in the single wires due to the perpendicular magnetic field. Studies $^{18}$ on low-resistance samples of the mean-field transition temperature showed a parabolic dependence on the magnetic field,

$$
T_{c}(H)=T_{c 0}-A H^{2} .
$$

Substituting Eq. (15) in Eq. (13) of $I_{c}(T)$, one finds that for $A H^{2} \ll<1, I_{c}(T)$ is proportional to $H^{2}$. As we will see

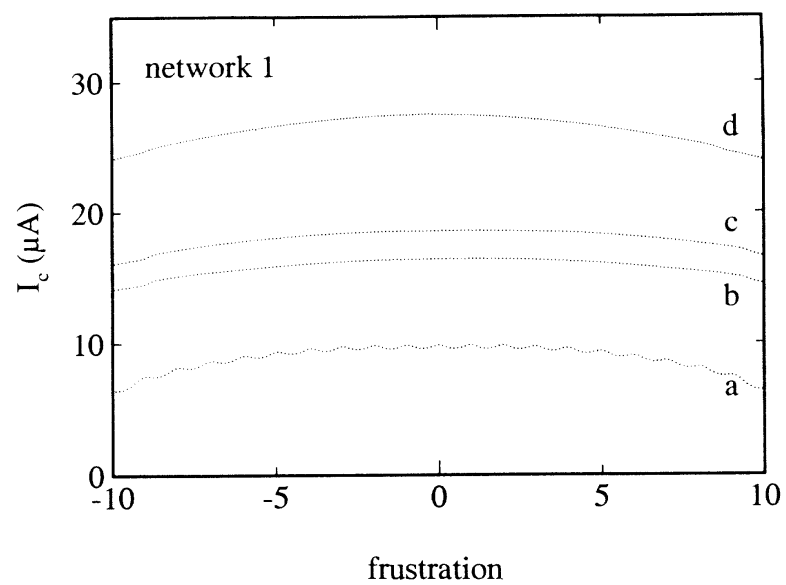

FIG. 7. Critical current of network 1 vs frustration for four different temperatures with (a) $T=2.05 \mathrm{~K}$, (b) $T=2.03 \mathrm{~K}$, (c) $T=2.02 \mathrm{~K}$, and (d) $T=1.94 \mathrm{~K}$.

in the next section, the vortex mobility is the dominant process in determining the network resistance. Substituting Eq. (15) in Eqs. (9) and (11), we also find ${ }^{19}$ a parabolic dependence of $R$ on $H^{2}$, and again this result is only valid for $A H^{2}<<1$. We have fitted the $I_{c}(f)$ of Fig. 7 and the $R(f)$ curves $d-g$ of Fig. 5 to this model of $T_{c 0}$ reduction with $A$ as an independent fitting parameter. For both the $I_{c}(f)$ and $R(f)$ curves, values of $A$ are obtained which lie between $1 \times 10^{-12}$ and $2 \times 10^{-12} \mathrm{~K} \mathrm{~m}^{2} \mathrm{~A}^{2}$. For a field of $0.1 \mathrm{~T}$, this value gives a reduction of $T_{c 0}$ with about 10 $\mathrm{mK}$. From the $R(f)$ curve, we find a zero-temperature thermodynamic critical field value of $2.10^{4} \mathrm{~A} / \mathrm{m}$ $\left(\mu_{0} H_{c}=0.03 \mathrm{~T}\right)$, which seems to be a reasonable value.

\section{Linear resistance}

In this section, we investigate the resistive transitions measured with a small applied current of $0.1 \mathrm{nA}(\mathrm{rms})$ per single wire. As a function of temperature, the $R(T)$ curve for network 1 is already given in Fig. 3. Here we will plot the data as a function of $\tau$ and $1 / \tau$. On a logarithmic scale in $R$, the zero-field result for the four networks is shown in Fig. 8.

As mentioned before, in zero magnetic field, twodimensional weakly coupled superconducting networks are expected to undergo a Kosterlitz-ThoulessBerezinskii phase transition. Just above the KTB transition temperature $\tau_{\mathrm{KTB}}$, the number of free vortices follows a square-root cusp dependence on temperature given $^{11,12}$ by

$$
S n_{f}(\tau)=c_{2} \exp \left\{-b\left(\tau-\tau_{\mathrm{KTB}}\right)^{-1 / 2}\right\} .
$$

For junction arrays in the temperature range $\tau>1$, the effective vortex mobility $\mu(\tau) \approx 1$ and the temperature dependence of the junction resistance is completely determined by the temperature dependence ${ }^{20}$ of $n_{f}(\tau)$ (dashed line in Fig. 8). The fact that the curves in Fig. 8 all lie orders of magnitude below the dashed line indicates that the vortex mobility in the wire networks is much lower than in junction arrays. The solid line in Fig. 8 is a fit to 


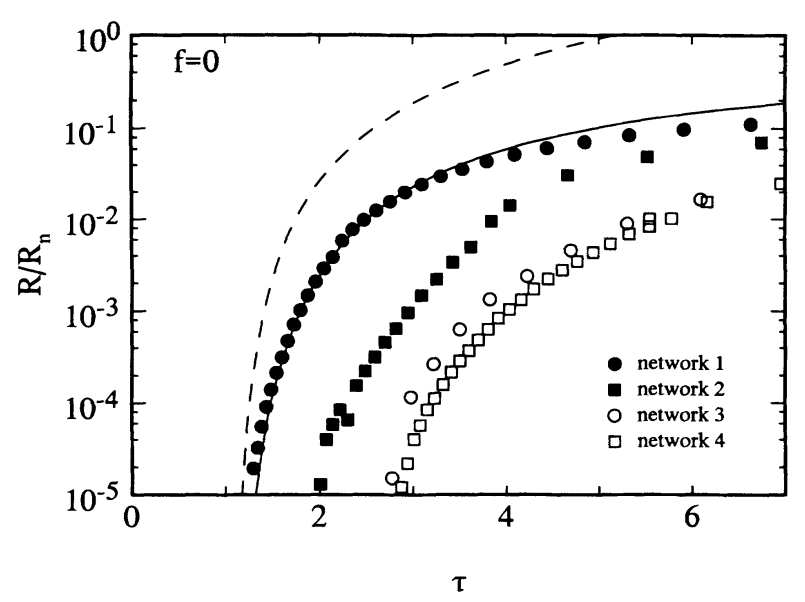

FIG. 8. Zero-field longitudinal resistive transitions of four networks as a function of normalized temperature $\tau$. The dashed line gives the universal prediction for the resistance in two-dimensional superconducting systems with an effecive vortex mobility $\mu(T) \approx 1$. The solid line is a fit to Eq. (16) with fit parameters $c_{3}=1.5, b=4.3$, and $\tau_{\mathrm{KTB}}=1.05$.

the square-root cusp behavior with reduced vortex mobility $\mu(\tau)$,

$$
\frac{R}{R_{n}}=c_{3} \exp \left\{-b\left(\tau-\tau_{\mathrm{KTB}}\right)^{-1 / 2}\right\} \exp \{-0.94 \xi(\tau) / s \tau\},
$$

with $c_{3}=1.5, b=4.3$, and $\tau_{\mathrm{KTB}}=1.05$. These values are all in reasonable agreement with the theoretical predictions. However, as argued before, ${ }^{4}$ this is a threeparameter fit, which leaves a certain degree of freedom. A more accurate test for the presence of a KTB transition is the nonlinear resistance, which will be discussed in the next section.

In Josephson junctions at low-resistance levels, the resistance deviates from the solid line and decreases exponentially. This is connected to single-vortex crossing over the array width. ${ }^{4}$ This process is a finite-size effect, and one expects to see similar behavior in wire networks. In Fig. 9 the zero-field resistance of network 1 is plotted as a function of $1 / \tau$. The solid line is the KTB fit of Eq. (16). At low-resistance levels, a deviation from the solid line is found and the resistance decreases exponentially. From the slope (dashed line), we find an energy bar-rier of $19 J(T)$. For an array with $W=100$, this yields a core energy of 6 in units of the coupling energy [Eq. (8)], which as expected is higher than the value of 4 in junction arrays.

In Fig. 9 we have also given the resistance for $f=0.15$. No exponential decrease as in junction arrays is found for this value of $f$. At low temperatures for this relatively high value of frustration, the number of vortices is determined by the applied field and is therefore temperature independent. Consequently, the transition at $f=0.15$ probes the temperature dependence of the vortex mobility, i.e., of $\exp \{-\xi(\tau) / \tau\}$, which goes as $\exp \left\{-t^{2 / 3} / T^{2}\right\}$.

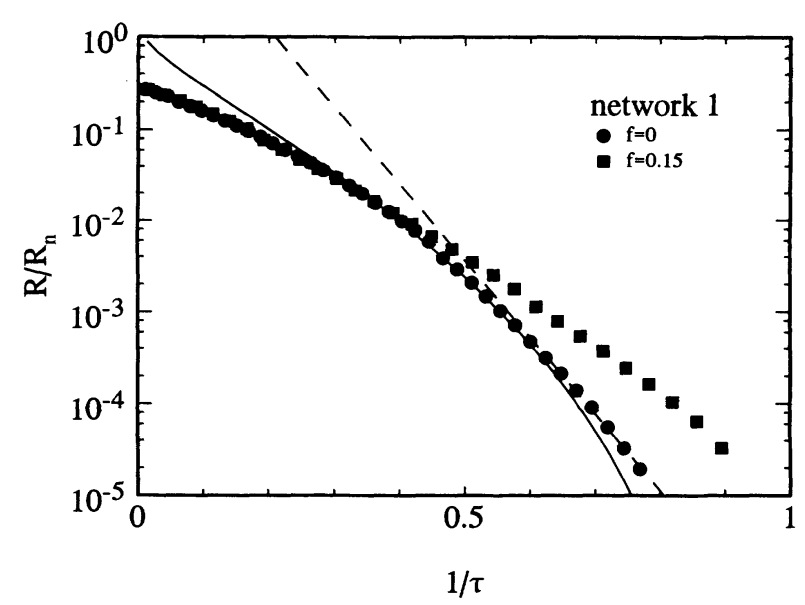

FIG. 9. Longitudinal normalized resistance as a function of inverse normalized temperature for two different frustrations. The solid line is the KTB fit to Eq. (16). The dashed line shows the exponential decrease at low temperatures, with an energy barrier of $19 J(T)$.

To check this, we have plotted the $\log _{10}(R)$ curves of Fig. 9 as a function of $t^{2 / 3} / T^{2}$. The result is given in Fig. 10 . High temperatures are found on the left-hand side of this plot. At lower temperatures, only for the data of $f=0.15$ a straight line is found. When plotted as $\log _{10}(R / R n)$ vs $t^{2 / 3} / T^{2}$, the slope should be $9.7 s / \sqrt{\xi_{0} l T_{c 0}}$. For network 1 , this number equals 229 . In Fig. 10 we find a slope of 254, in good agreement.

The zero-field transitions of the other arrays lie more than an order of magnitude below the one of network 1 . The vortex mobility in these networks is greatly suppressed and probably the resistance shows a temperature dependence of $t^{2 / 3} / T^{2}$ rather than the square-root cusp dependence expected for a KTB transition. We plotted these transitions as a function of $t^{2 / 3} / T^{2}$ and found straight lines with measured (calculated) values of the slopes $1450(180)$ for network $2,1000(840)$ for network 3, and 1070 (650) for network 4. We do not under-

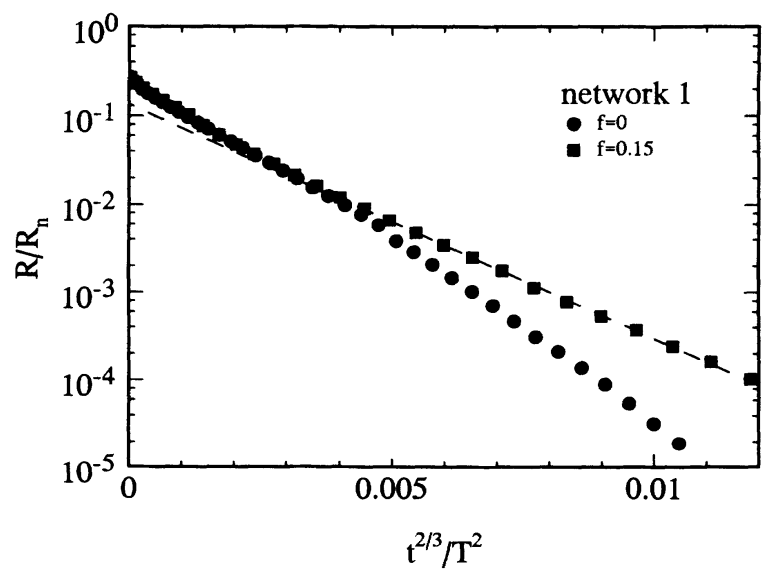

FIG. 10. Longitudinal normalized resistance plotted as a function of $t^{2 / 3} / T^{2}$, which is the temperature dependence of the effective vortex mobility [Eq. (11)]. The dashed line is a fit through the data of $f=0.15$ with a slope of 254 . 
stand the large discrepancy for network 2. Even if we take into account that the single wires are not one dimensional [the $w / \xi(T)$ ratio varies between 2 and 4 for the temperatures of interest], this seems to be a much too large value.

\section{Nonlinear resistance}

With the nonlinear resistance measurement, the presence of a KTB transition can be determined in an accurate way. The transport current through the array is varied while the voltage across the array is measured. Various $I-V$ curves are taken at temperatures close to the KTB transition temperature. When plotted as $\ln V$ vs $\ln I$, above the transition the $I-V$ characteristics show curvature, with slopes close to 1 at the lowest-current levels. This is the regime where free thermally generated vortices move across the sample. Below the transition, the $I-V$ curves show a power law dependence $V \sim I^{a(\tau)}$, which is connected to current-induced vortex pair unbinding. At the transition the nonlinear resistance exponent $a(\tau)$ is expected to jump from 3 (just below the transition) to 1 (just above the transition). The jump from 3 to 1 has a universal value and corresponds to the jump in the superfiuid density. ${ }^{21}$ At low temperatures, the exponent is proportional to $1 / \tau, a(\tau)-1=\pi / \tau$.

For various temperatures, we have measured the $\ln V-\ln I$ plots for network 1 at $f=0$ and 0.05 and for network 2 at $f=0$. We have determined $a(\tau)$ at a voltage level of $50 \mathrm{nV}$. The result for network 1 is shown in Fig. 11. For the zero-field data, a clear jump is observed, close to the universal value of 3 . The transition takes place at $\tau_{\mathrm{KTB}}=1$, the same value as found from the fit of the linear resistance to the square-root cusp behavior. In Monte Carlo (MC) computer simulations of the helicity modulus $\Gamma$, a measure of the phase correlations in the $X Y$ model, the transition lies ${ }^{22}$ at $\tau_{\mathrm{KTB}}=0.95$. Our experimental value lies very close to this value obtained from the MC calculation. For low temperatures (right-hand side of the plot), $a(\tau)$ is proportional to $1 / \tau$. The slope is 3.5 , somewhat higher than the expected value $\pi$. For the data taken at $f=0.05$, clearly no jump is seen and there is, as expected, no KTB transition at this value of the frustration.

For network 2, we again derived the nonlinear resistance exponent from $\ln V-\ln I$ plots at a voltage level of 50 $\mathrm{nV}$ and plotted the result as a function of $1 / \tau$. For this network, no clear jump is observed. At this moment we do not have an explanation for this, but it is consistent with the observation in the linear resistance. There we found a much larger barrier than expected, indicating that the vortices are effectively pinned in the periodic network potential. When we determine the energy barrier for network 2 from the linear resistance measurement, we find a barrier of about $48 J(T)$ at the transition temperature. This number is much larger than the energy of a vortex pair with maximum separation $\left(\pi^{2}+2 \pi \ln \{W / 2\}\right) J(T)$, which equals $34 J(T)$ for a network with $W=100$. Consequently, no current induced pair unbinding is expected to be observed in the nonlinear resistance measurement.

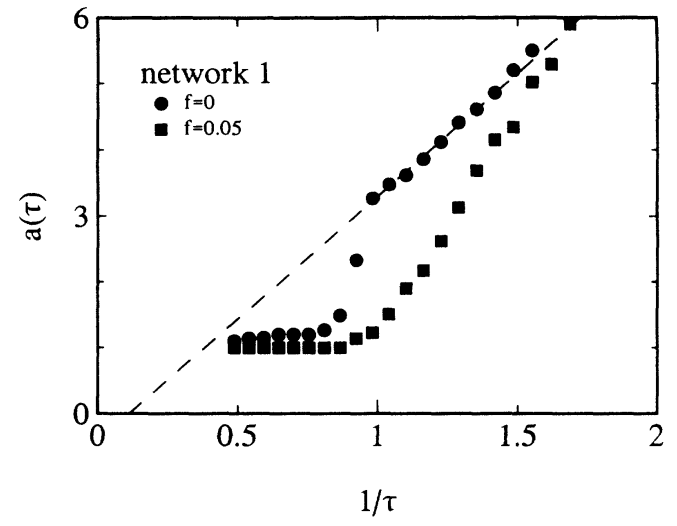

FIG. 11. Nonlinear resistance exponent of current-voltage characteristics as a function of inverse normalized temperature for two values of $f$, showing a KTB transition with a universal jump for $f=0$ at $\tau \approx 1$. For $f=0$ at lower temperatures, the exponent is proportional to $1 / \tau$ with a slope of 3.5 (dashed line).

\section{DISCUSSION}

\section{A. Resistive behavior}

We have shown data on four different networks, two with $s=500 \mathrm{~nm}$ and two with $s=2000 \mathrm{~nm}$. The networks with $s=2000 \mathrm{~nm}$ do not show oscillating behavior of the resistance as a function of $f$, whereas for the networks with $s=500 \mathrm{~nm}$ clear oscillations are observed. The most detailed measurements were performed on network 1 with $s=500 \mathrm{~nm}$. This network has the smallest wire width $w=100 \mathrm{~nm}$, which for the temperatures of interest is of the order of the Ginzburg-Landau coherence length $\xi(T)$.

For this network in zero field, a clear KosterlitzThouless-Berezinskii phase transition has been observed with a universal jump of the nonlinear resistance exponent at the transition. The transition takes place at the same normalized temperature as found in junction arrays ${ }^{4}$ and in Monte Carlo simulations. ${ }^{22}$ We have fitted the temperature dependence of the linear resistance to the theoretical square-root cusp prediction, and a good fit could only be obtained by taking into account the lower vortex mobility in the wire networks. At the lowestresistance levels, however, the linear resistance decreases exponentially with inverse normalized temperature $\tau$, indicating thermally activated vortex flow. In zero field we find an energy barrier $19 J(T)$ close to the predicted value for single-vortex crossings over the whole width of the sample [see Eq. (8)].

For $f=0.15$ at temperatures below the KTB transition temperature, only magnetically induced vortices are present. Since their number is fixed, the resistive transition at $f=0.15$ probes the temperature dependence of the vortex mobility [Eq. (7)]. The vortex mobility is determined by thermal activation over the energy barrier, which a vortex has to overcome when moving from one cell to the next. In wire networks there are two mechanisms determining this barrier. One, as in junction arrays, is the energy associated with the phase differences in 
the network. The other contribution is due to the loss of condensation energy when the vortex crosses the wire and is the dominant one in our networks. In the measurements, we indeed find that the resistance at $f=0.15$ follows the temperature dependence of the vortex mobility and that the height of the energy barrier is close to the loss of condensation energy in a one-dimensional wire. Expressed in units of the coupling energy $J(T)$, this barrier is equal to $s / \xi(T)$.

In our experiments we find that a greatly reduced vortex mobility masks the observation of a KTB transition and suppresses the resistance oscillations in a field. By considering the two different energy barriers for vortex motion in 2D networks, one finds that the $s / \xi(T)$ ratio is the determining factor for observation of a KTB transition and of oscillations in the magnetoresistance. At the lowest temperatures, the linear resistance decreases exponentially for all $f$. In zero field, there are single-vortex crossings over the whole array width characterized by an energy barrier $U_{2 \mathrm{D}} \approx\{4+\pi \ln (2 W / \pi)\} J(T)$. Above the entry field $f \approx 1 / W,{ }^{4}$ the relevant energy barrier is the field-independent barrier for crossing from one cell to the next, $U_{1 \mathrm{D}} \approx\{s / \xi(T)\} J(T)$. Roughly speaking, resistance oscillations and the square-root cusp dependence characteristic of a KTB transition can be seen when $U_{2 \mathrm{D}}>U_{1 \mathrm{D}}$, i.e., when

$$
\frac{s}{\xi(T)}<4+\pi \ln (2 W / \pi) \text {. }
$$

For our networks with $s=2000 \mathrm{~nm}$, the $s / \xi(T)$ ratio is so large for the temperatures of interest that vortices are effectively pinned in the network and that the vortex mobility dominates the resistive behavior of all $f$.

\section{B. Critical current versus magnetic field}

In this section we discuss the surprising result that at low temperatures the critical current of network 1 does not oscillate as a function of applied field, whereas at the same temperatures small oscillations are found in the resistance. Only at high temperatures close to $T_{c 0}$, are oscillations in the critical current found. For network 1, critical current oscillations are observed above $2.04 \mathrm{~K}$, which is $95 \mathrm{mK}$ below $T_{c 0}$. This is in contrast with junction arrays, where resistance oscillations are always connected to oscillations in the critical current.

For wire networks, the dependence of the supercurrent on the phase difference across adjacent nodes is given in Eq. (5). One expects the critical current oscillations to become smaller when temperature decreases because $\xi(T)$ and therefore $\Delta \phi_{\max }$ decrease. However, this effect cannot explain the absence of critical current oscillations in the experiment. Consider, e.g., $f=\frac{1}{2}$. At $f=\frac{1}{2}$ the ground state ${ }^{23}$ is a pattern of circulating supercurrents with amplitude $i_{c} \pi /\left(4 \Delta \phi_{\max }\right)$. This pattern is shown in Fig. 12(a). The $A_{i j}$ 's are chosen to be zero on horizontal bonds and $A_{i j}=2 \pi f y$ on vertical bonds with periodic boundary conditions in the $x$ direction. The maximum possible current per bond that can be transported in the $y$ direction ${ }^{24}$ is $i_{c}(T)\left\{\Delta \phi_{\max }-\pi / 4\right\} / \Delta \phi_{\max }$. In network 1 at $100 \mathrm{mK}$ below $T_{c 0}, \Delta \phi_{\max }=2.7$ and the maximum
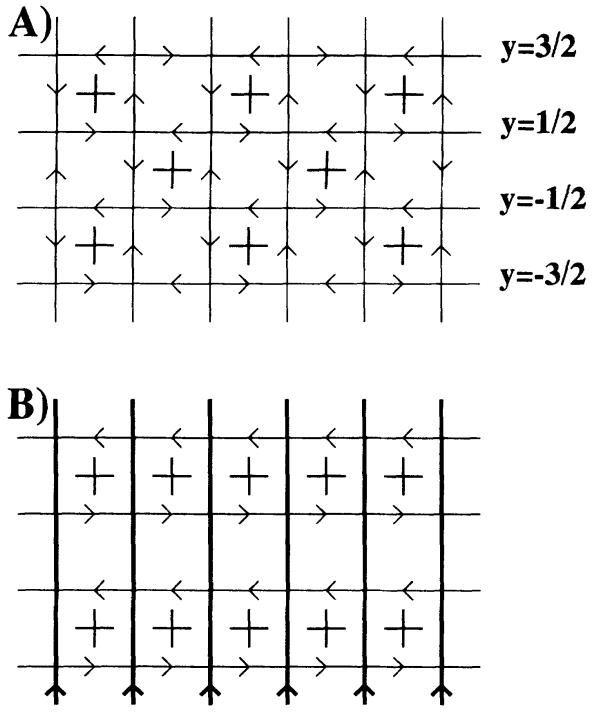

FIG. 12. Schematic drawings of supercurrent configurations in a wire network at $f=1 / 2$. The arrows indicate the direction of the supercurrents, the crosses the vortex-charge configuration. The $y$ axis is in the vertical direction, the $x$ axis in the horizontal direction. In (a) the "checkerboard" configuration is shown, which has the lowest energy for zero applied current. In (b) a vortex configuration is shown which can carry the maximum critical current.

current per bond that can be transported through the network is $25 \%$ smaller than the current that can be transported at $f=0$. In the experiment, however, we find that the maximum transported currents at $f=0$ and $\frac{1}{2}$ are about equal.

We believe that the absence of critical current oscillations has to be sought in the linear current-phase relation of wire networks with $s \gg \xi(T)$. As an example, consider Fig. 12(b), which shows a vortex configuration for $f=\frac{1}{2}$ when a current is applied in the $y$ direction. The key to this configuration is that the phases of the islands on each horizontal row have the same value so that the maximum critical current can flow in the $y$ direction. The currents in the $x$ direction are solely due to the $A_{i j}$ 's; i.e., a supercurrent of $i_{c} \pi /\left(2 \Delta \phi_{\max }\right)$ flows in the horizontal direction. In junction arrays a phase difference of $\pi / 2$ always corresponds to the critical current, and therefore the mechanism for depinning of the vortex lattice may well be different in wire networks. Our model, of course, relies on strong simplifications as there are no periodic boundary conditions in our measured networks and some of the currents induced by the $A_{i j}$ 's have to flow at the edges parallel to the transport current. Furthermore, there are thermally activated vortex hopping events out of potential wells formed by the intrinsic pinning of the network accounting for the observed small frustration dependent (zero-bias) resistance. A more detailed study is needed to reveal the exact mechanism for depinning of the vortex lattice in wire networks which should include the linear current-phase relation and should consider other values of $f$. In this light it is worth mentioning that recently the 
vortex configuration of networks in a magnetic field have been mapped out, ${ }^{25-27}$ but no transport current was applied. It would be interesting to repeat the measurements in weakly coupled wire networks at low enough temperatures so that vortex hopping does not play a role, to visualize the depinning of the vortex lattice in wire networks with a linear current phase relation.

\section{CONCLUSIONS}

We have fabricated superconducting weakly coupled wire networks, consisting of wires with a width in the order of and a length $s$ much larger than the GinzburgLandau coherence length $\xi(T)$. The ratio $s / \xi(T)$ determines the barrier height for a vortex to cross from one cell to the next. If this ratio is not too large, the resistive behavior is qualitatively similar to that observed in Josephson-junction arrays, including a KosterlitzThouless-Berezinskii transition with a universal jump for $f=0$ and resistance oscillations as a function of applied magnetic field. The vortex mobility in wire networks is, however, much lower than in junction arrays. Wire networks with $s \gg \xi(T)$ have a linear current-phase relation and the maximum phase difference across adjacent nodes can be much larger than $\pi / 2$. As a consequence, vortex configurations can exist which differ from the ones in junction arrays, which may explain our observation that at low temperatures the critical current in a wire network does not oscillate as a function of applied magnetic field.

\section{ACKNOWLEDGMENTS}

Samples were made at the Delft Institute of Micro Electronics and Submicron Technology (DIMES). We thank L. E. M. de Groot and B. G. M. de Lange for $e$ beam lithography, J. Toth for his assistance in making SEM micrographs, and H. W. Zandbergen of the National Center for HREM at the Delft University of Technology for making the high-resolution TEM micrographs of the niobium-silicon films. One of us (H.v.d.Z.) expresses his gratitude to P. Martinoli and B. Pannetier for their hospitality during visits to their group. Part of this work was supported by the Dutch Foundation for Fundamental Research on Matter (FOM). H.v.d.Z also acknowledges support from the NSF through Grant No. DMR9108748.
*Present address: MIT, Cambridge, MA 02139.

${ }^{\dagger}$ Present address: DIMES, Delft University of Technology, The Netherlands.

${ }^{1}$ For a review of this field, see Proceedings of the Nato Advanced Research Workshop on Coherence in Superconducting Networks, Delft, 1987, edited by J. E. Mooij and G. Schön, Physica B 152, 1 (1988).

${ }^{2}$ V. L. Berezinskii, Zh. Eksp. Teor. Fiz. 59, 907 (1970) [Sov. Phys. JETP 32, 493 (1971)]; J. M. Kosterlitz and D. J. Thouless, J. Phys. C 6, 1181 (1973).

${ }^{3}$ See, for a review, J. E. Mooij, in Percolation, Localization, and Superconductivity, edited by A. M. Goldman and S. A. Wolf (Plenum, New York, 1983), p. 325.

${ }^{4}$ H. S. J. van der Zant, H. A. Rijken, and J. E. Mooij, J. Low Temp. Phys. 79, 289 (1990).

${ }^{5}$ H. S. J. van der Zant, H. A. Rijken, and J. E. Mooij, J. Low Temp. Phys. 82, 62 (1991).

${ }^{6}$ B. Pannetier, J. Chaussy, and R. Rammal, Jpn. J. Appl. Phys. 26, Suppl. 26-3, 1994 (1987), and references therein.

${ }^{7}$ B. Jeanneret, Ph. Fluckiger, Ch. Leeman, and P. Martinoli, IEEE Trans. Magn. MAG-25, 1428 (1989); B. Jeanneret, Ph. Fluckiger, J. L. Gavilano, Ch. Leeman, and P. Martinoli, Phys. Rev. B 40, 11374 (1989).

${ }^{8}$ H. S. J. van der Zant, M. N. Webster, J. Romijn, and J. E. Mooij, Phys. Rev. B 42, 2647 (1990); Physica B 165\&166, 1635 (1990).

${ }^{9}$ M. Giroud, O. Buisson, Y. Y. Wang, B. Pannetier, and D. Mailly, J. Low Temp. Phys. 87, 683 (1992).

${ }^{10}$ S. Teitel and C. Jayaprakash, J. Phys. (Paris) Lett. 46, L33 (1985).

${ }^{11}$ B. I. Halperin and D. R. Nelson, J. Low Temp. Phys. 36, 599 (1979).

${ }^{12}$ C. J. Lobb, D. W. Abraham, and M. Tinkham, Phys. Rev. B 27, 150 (1983).

${ }^{13}$ M. Tinkham, Introduction to Superconductivity (McGrawHill, New York, 1975), Chap. 7.

${ }^{14}$ W. L. Johnson, C. C. Tsuei, S. T. Raider, and R. B. Laibowitz,
J. Appl. Phys. 50, 4241 (1979).

${ }^{15}$ H. S. J. van der Zant, J. F. Jongste, H. W. Zandbergen, and J. E. Mooij, Physica B 165\&166, 1525 (1990).

${ }^{16}$ L. G. Aslamazov and A. I. Larkin, Phys. Lett. 26A, 238 (1968).

${ }^{17}$ A. T. Fiory, A. F. Hebard, and W. I. Glaberson, Phys. Rev. B 28, 5075 (1983).

${ }^{18}$ B. Pannetier, J. Chaussy, R. Rammel, and J. C. Villegier, Phys. Rev. Lett. 53, 1845 (1984).

${ }^{19}$ The temperature-dependent thermodynamic critical field is $H_{c}(T, H)=1.73 H_{c}(0)\left\{T_{c}(H)-T\right\} / T_{c}(H)$. With Eq. (15), this gives $H_{c}(T, H)=1.73 H_{c}(0)\left\{1-T / T_{c 0}-A H^{2}(2+T /\right.$ $\left.\left.T_{c 0}\right) / T_{c 0}\right\}$. Substituting this in Eq. (9), one finds that $U_{1 \mathrm{D}} \propto 1.73 H_{c}(0)\left(1-T / T_{c 0}\right)^{2}\left\{1-2 A H^{2}\left(2 T_{c 0}+T\right) /\left[T_{c 0}\left(T_{c 0}\right.\right.\right.$ $-T)]\}$. Using $\exp \{-(1-x)\} \approx x-1$ for small $x$, one sees that the vortex mobility is proportional to $H^{2}$.

${ }^{20}$ The dashed line in Fig. 8 is the universal prediction for resistance in two-dimensional superconducting systems, with $\mu(T) \approx 1$ [see Minnhagen, Rev. Mod. Phys. 59, 1001 (1987)]. For $T>T_{\mathrm{KTB}}$, Josephson-junction arrays and thin films have $\mu(T) \approx 1$. Experimental results are in good agreement with this universal prediction [for junction arrays, see Ref. 4; for thin films, see J. C. Garland and H. J. Lee, Phys. Rev. B 36, $3638(1987)]$.

${ }^{21}$ D. R. Nelson and J. R. Kosterlitz, Phys. Rev. Lett. 39, 1201 (1977).

${ }^{22}$ S. Teitel and C. Jayaprakash, Phys. Rev. B 27, 598 (1983).

${ }^{23}$ T. C. Halsey, J. Phys. C 18, 2437 (1985).

${ }^{24}$ S. P. Benz, M. S. Rzchowski, M. Tinkham, and C. J. Lobb, Phys. Rev. B 42, 6165 (1990).

${ }^{25}$ L. N. Vu, M. S. Wistrom, and D. J. van Harlingen, Appl. Phys. Lett. 63, 1693 (1993).

${ }^{26}$ H. D. Hallen, R. R. Seshadri, A. M. Chang, R. E. Miller, L. N. Pfeiffer, K. W. West, C. A. Murray, and H. F. Hess, Phys. Rev. Lett. 71, 3007 (1993).

${ }^{27} \mathrm{~K}$. Runge and B. Pannetier (private communication). 


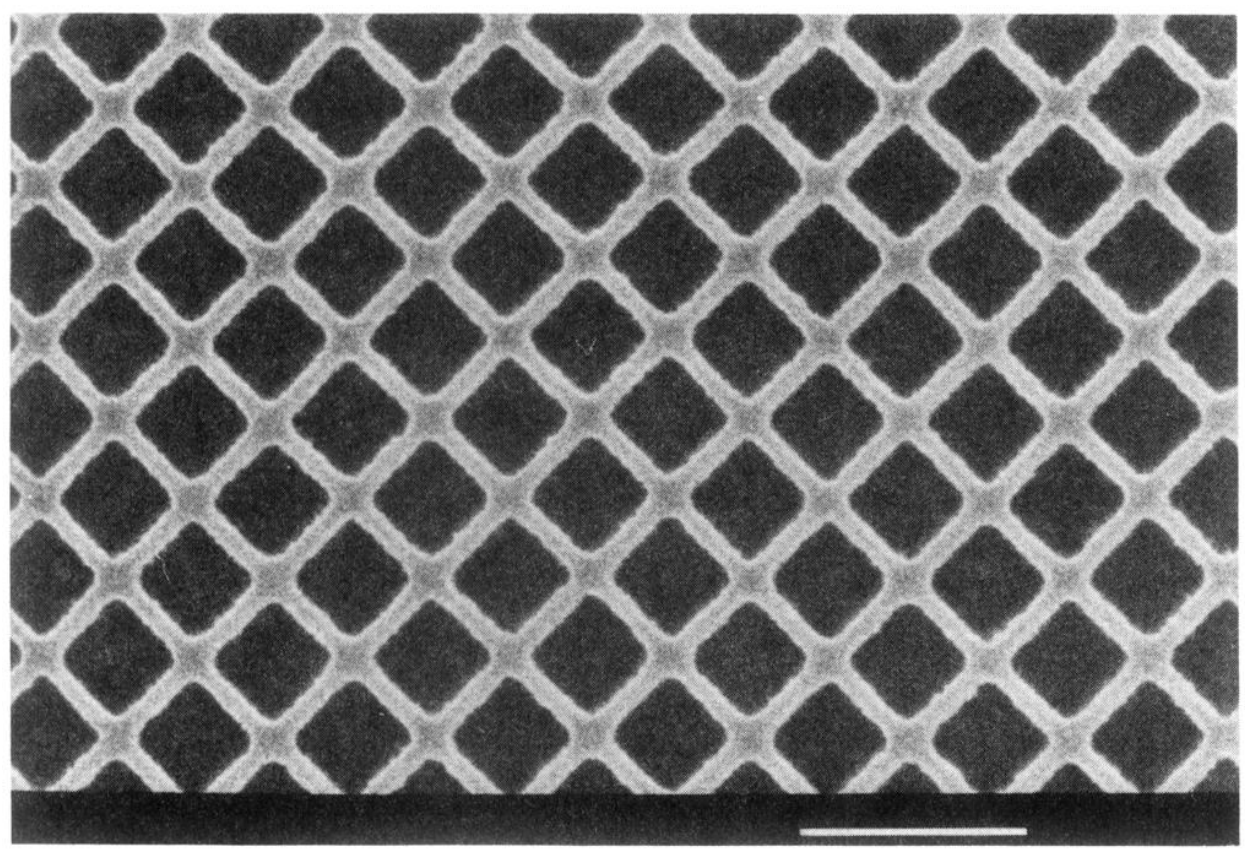

FIG. 1. Scanning electron microscope (SEM) micrograph of network 1 . The white bar at the bottom indicates $1 \mu \mathrm{m}$. The width of the single wires is about $100 \mathrm{~nm}$; the lattice spacing $s$ is $500 \mathrm{~nm}$. 


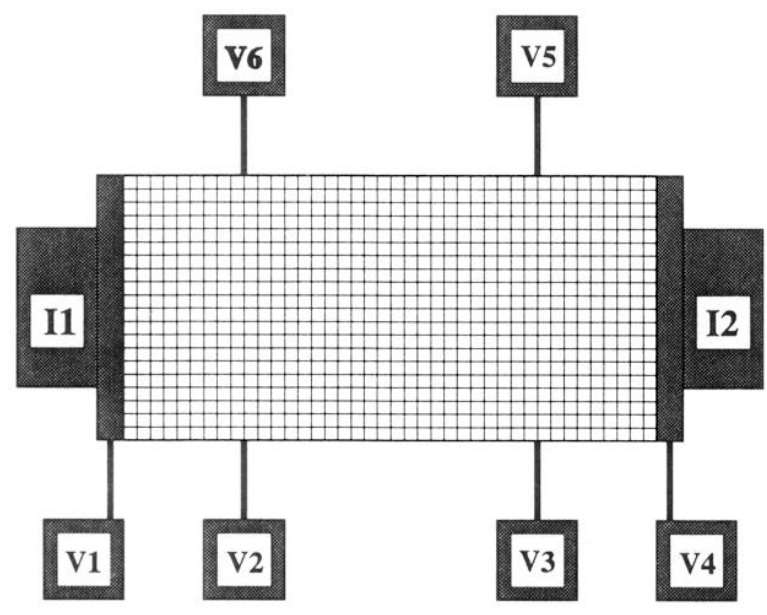

FIG. 2. Schematic drawing of a wire network. 